Category: Optoelectronics

\title{
Laser Molecular Beam Epitaxy Growth of GaN layer on Sapphire (0001) under various process conditions
}

\author{
Sunil S. Kushvaha, M. Senthil Kumar, Bipin K. Gupta, and K. K. Maurya \\ CSIR-National Physical Laboratory, Dr. K.S. Krishnan Road, New Delhi-110012, India \\ E-mail: kushvahas@nplindia.org
}

\begin{abstract}
We have grown high quality epitaxial GaN films on sapphire (0001) substrates using a ultra-high vacuum laser molecular beam epitaxy (MBE) system at different growth temperatures, deposition rate and nitrogen species. The HVPE grown GaN solid target was ablated at laser energy density $\sim 5$ $\mathrm{J} / \mathrm{cm}^{2}$ with laser frequency $\sim 5 \mathrm{~Hz}$ (low flux) and $10 \mathrm{~Hz}$ (high flux) in presence of r.f. nitrogen plasma. Structural properties of the epitaxial GaN films were characterized using high resolution $x$-ray diffraction, atomic force microscopy (AFM), and photoluminescence spectroscopy (PL). At high flux, the full width at half maximum (FWHM) of x-ray diffraction rocking curve of GaN (0002) peak decreases with increasing growth temperature from 500 to $720{ }^{\circ} \mathrm{C}$. The $\mathrm{GaN}$ film grown at $700{ }^{\circ} \mathrm{C}$ with low flux shows a large FWHM (368 arc sec) with small grain sizes in comparison to the GaN film grown with high flux (FWHM: 110 arc sec). We have also studied the effect of high pressure nitrogen ambient during ablation of GaN target for growth of GaN films on sapphire with and without prenitridation of sapphire at growth temperature $500{ }^{\circ} \mathrm{C}$. The typical PL measurement on the GaN film grown on sapphire using laser MBE system shows the high quality of GaN film with minimum defects. The obtained results suggest that the present growth technique could be an alternative for fabrication of high quality GaN based devices.
\end{abstract}

Index Terms - GaN, Laser Molecular Beam Epitaxy, Sapphire, High resolution x-ray diffraction, Atomic Force Microscopy, Photoluminescence.

\section{INTRODUCTION}

The III-N based semiconductor materials have been attracting immense interest particularly due to their key role in many device applications such as ultraviolet light emitting diodes, high electron mobility transistors, optical data storage and related devices. ${ }^{1-3}$ The structural and optical properties of $\mathrm{GaN}$ films are the key factors for the realization of high performance $\mathrm{GaN}$ based devices. The GaN films are mainly grown by epitaxial techniques such as hydride vapor phase epitaxy (HVPE), ${ }^{4}$ metal organic chemical vapor deposition (MOCVD), ${ }^{5,6}$ plasma assisted molecular beam epitaxy (PA$\mathrm{MBE})^{7}$ and laser molecular beam epitaxy (LMBE) ${ }^{8}$ However, the HVPE and MOCVD techniques employ a high growth temperature for the growth of $\mathrm{GaN}$ layers. The high growth temperature may lead to formation of unwanted compounds or alloys at interfaces, which may degrade and affect the device performance. In this regard, a low temperature growth will be beneficial and exceptional alternate because there is a low probability of formation of unwanted interfacial compounds or alloys at interface. The LMBE technique has advantage that the growth of $\mathrm{GaN}$ film can occur at a low growth temperature due to high-kinetic energy film precursors produced by laser ablation of targets. ${ }^{8}$

Here, we report the growth of high crystalline quality epitaxial $\mathrm{GaN}$ films on pre-nitridated sapphire (0001) substrates by ablating the HVPE grown high pure solid GaN target with different flux in presence of r.f. nitrogen plasma. The effects of growth temperature, nitrogen source and buffer layer on the structural properties of the epitaxial GaN films have been also studied in details.

\section{EXPERIMENTAL DETAILS}

The epitaxial GaN films were grown on pre-nitridated sapphire (0001) substrates using an ultra-high vacuum (UHV) laser MBE system (SVT Associates, Inc. US). The laser MBE system consists two chambers: main growth chamber and preparation cum load-lock chamber. The main growth chamber is equipped with reflection high energy electron diffraction (RHEED), laser ablation targets, infrared radiation heating substrate stage and a radio-frequency (RF) nitrogen plasma source to supply nitrogen radicals. The base pressure in the GaN growth chamber was better than $2 \times 10^{-10}$ Torr. The details of sapphire cleaning and nitridation in laser MBE system has been discussed previously. ${ }^{9}$ A high purity polycrystalline HVPE grown GaN target $(99.9999 \%)$ was ablated using a $\mathrm{KrF}$ excimer laser (248-nm wavelength, $25 \mathrm{~ns}$ pulse) with an energy density of $\sim 5 \mathrm{~J} / \mathrm{cm}^{2}$ at different repetition rates $(5 \mathrm{~Hz}$ and $10 \mathrm{~Hz}$ ). Generally, laser ablated $\mathrm{GaN}$ plume consists the energetic species such as $\mathrm{GaN}$, nitrogen deficient $\mathrm{GaN}_{1-\mathrm{x}}$ and $\mathrm{Ga}$ and the grown $\mathrm{GaN}$ samples have deficiency in nitrogen. ${ }^{8}$ To overcome with nitrogen deficiency, the nitrogen radicals or high pressure nitrogen gas were supplied for stoichiometric GaN growth.

The cleaning of sapphire substrate, surface nitridation and growth of GaN film were monitored in-situ using the RHEED. The laser MBE grown GaN films were ex-situ characterized using various techniques such as high-resolution $\mathrm{x}$-ray diffraction (HR-XRD), atomic force microscopy (AFM) and photoluminescence (PL) spectroscopy. The Multimode-V Veeco AFM was employed in tapping mode to characterize the surface morphology of GaN films using AFM tips of curvature radii less than $10 \mathrm{~nm}$. The root-mean-square (rms) roughness of $\mathrm{GaN}$ surfaces was determined from AFM images of $2 \mu \mathrm{m} \times 2 \mu \mathrm{m}$ areas. The PL measurements of the GaN samples were performed with excitation wavelength of 325 $\mathrm{nm}$ generated by a Xenon lamp filtered by an excitation monochromator. The beam was focused onto the sample 
mounted at $45^{\circ}$ to the incident light. The emitted PL signals were passed through an emission monochromator and recorded using a photomultiplier tube.

\section{RESULTS \& DISCUSSIONS}

The GaN epitaxial layers have been grown on c-plane pre-nitridated sapphire substrates in the growth temperature range $500-720^{\circ} \mathrm{C}$ at high flux. The XRD $2 \theta$ scan of GaN film grown on sapphire by laser MBE showed only $\{0001\}$ family of $\mathrm{GaN}$ and sapphire. The phi-scan XRD data also confirmed the hexagonal structure of $\mathrm{GaN}$ on sapphire with $30^{\circ}$ rotation of GaN unit cell with respect to sapphire. ${ }^{9}$ The x-ray rocking curves of $\mathrm{GaN}$ films were measured for (0002) $\mathrm{GaN}$ plane. The FWHM of GaN (0002) peak decreases from 1582 arc sec to 102 arc sec with increase of growth temperature from 500 to $720{ }^{\circ} \mathrm{C}$. A x-ray rocking curve FWHM of 110-102 arc sec [Fig. 1] was obtained for growth temperature $700-720{ }^{\circ} \mathrm{C}$, which is lower than the previously reported value of 420 arc sec for $\mathrm{GaN}$ film on sapphire grown using a polycrystalline GaN solid target. ${ }^{8}$ The AFM studies also showed that the average grain sizes of the $\mathrm{GaN}$ film increases with increasing growth temperature from $500{ }^{\circ} \mathrm{C}$ to $720{ }^{\circ} \mathrm{C}$ at high growth rate as shown in Fig. 2(a-b). Fig. 2(a) shows the surface morphology of the GaN film grown at $600{ }^{\circ} \mathrm{C}$ of grain size $80-85 \mathrm{~nm}$. With further increase in growth temperature (700 $\left.{ }^{\circ} \mathrm{C}\right)$, mostly large grains ( $\left.200 \mathrm{~nm}-300 \mathrm{~nm}\right)$ of GaN films with flat facets were obtained, indicating the enhanced growth along lateral direction as seen in Fig. 2(b).

The quality of $\mathrm{GaN}$ film grown at low flux (laser parameters: energy density $5 \mathrm{~J} / \mathrm{cm}^{2}$, frequency $5 \mathrm{~Hz}$ ) with presence of nitrogen radical supplied through r.f. plasma at growth temperature $700{ }^{\circ} \mathrm{C}$ was also characterized with HR$\mathrm{XRD}$ and AFM. Figure 1 represents the normalized $\mathrm{X}$-ray rocking curves of $\mathrm{GaN}(0002)$ plane grown at $700{ }^{\circ} \mathrm{C}$ with low and high fluxes. It was found that the FWHM value of $\mathrm{GaN}(002)$ peak is large (368 arc sec) for the GaN grown at low growth rate compared to $\mathrm{GaN}$ film grown with high growth rate (110 arc sec). The grain size of GaN film grown at low flux rate is random and it varies from $80 \mathrm{~nm}$ to $300 \mathrm{~nm}$ as shown in Fig. 2(c). From AFM and HR-XRD analysis, it is clear that the low flux is not favorable to grow high quality smooth GaN film at growth temperature of $700{ }^{\circ} \mathrm{C}$.

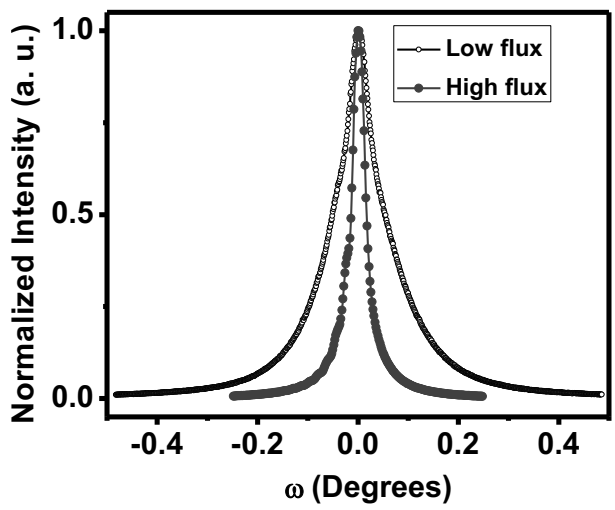

Fig. 1: Normalized x-ray rocking curves of GaN (0002) plane grown at $700{ }^{\circ} \mathrm{C}$ with low and high flux.
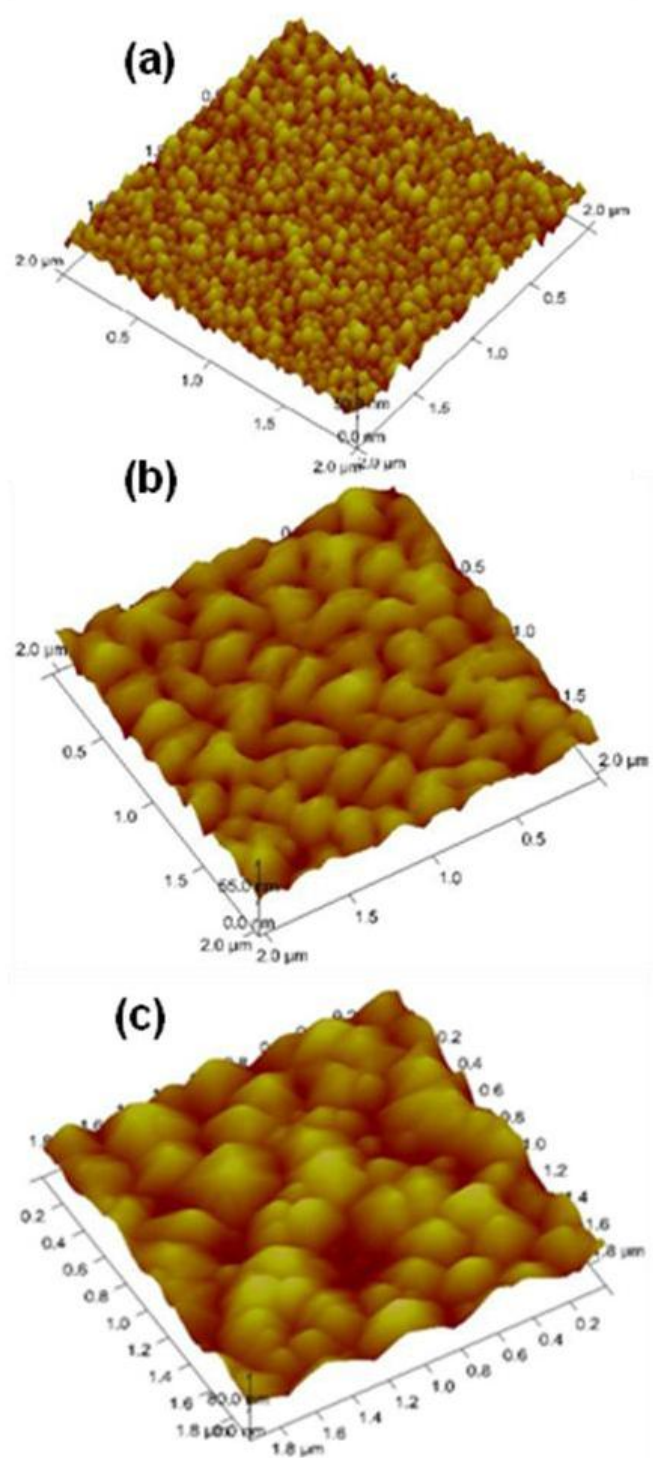

Fig. 2: AFM images of GaN films grown on sapphire at different growth temperatures: (a) $600{ }^{\circ} \mathrm{C}$, (b) $700{ }^{\circ} \mathrm{C}$ with high flux. (c) AFM image of $\mathrm{GaN}$ film grown at $700{ }^{\circ} \mathrm{C}$ at low flux.

The effect of high pressure nitrogen environment during laser ablation of bulk GaN target on the quality of $\mathrm{GaN}$ film grown with and without pre-nitridation of sapphire at growth temperature $500{ }^{\circ} \mathrm{C}$ has also been studied. The FWHM of $\mathrm{x}-$ ray rocking curves measured for $\mathrm{GaN}$ (0002) plane of high pressure grown $\mathrm{GaN}$ layer on bare-sapphire is larger (720 arc $\mathrm{sec})$ than that on pre-nitridated sapphire (218 arc sec) as shown in Fig. 3. The AFM studies showed the variation in surface morphology and rms roughness at different growth conditions as shown in Fig. 3(a-b). The large and flat grains $(150-250 \mathrm{~nm})$ of $\mathrm{GaN}$ film was obtained on pre-nitridated sapphire with rms surface roughness of $2 \mathrm{~nm}$ [Fig 4(a)]. Without pre-nitridation of sapphire substrate, the grown GaN film has a RMS surface roughness of $11 \mathrm{~nm}$ with grain sizes of 100-150 nm as shown in Fig. 4(b). The AFM and HR-XRD measurements revealed that the pre-nitridation of sapphire substrates using nitrogen radicals acts as an intermediate or 
buffer layer for growing a high quality smooth surface GaN film. $^{10}$

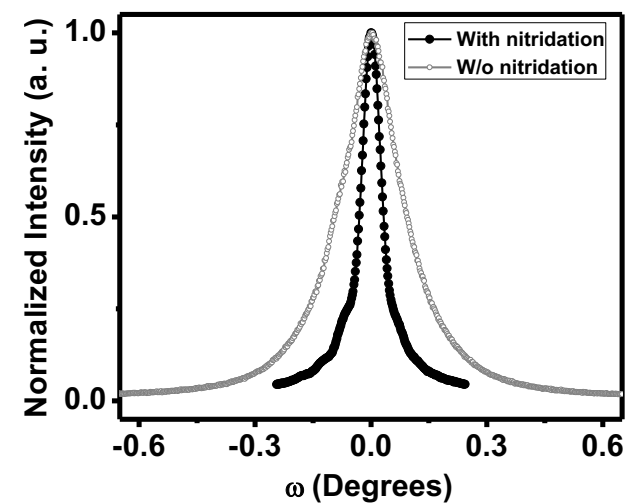

Fig. 3: Normalized x-ray rocking curves of $\mathrm{GaN}(0002)$ plane grown in presence of high pressure nitrogen at $500{ }^{\circ} \mathrm{C}$ with nitridation and without nitridation on sapphire.

It has been observed that the epitaxial GaN films grown on sapphire exhibit a high dislocation density. The xray rocking curves measured for different planes have been widely used indirectly to analyze the presence of dislocation in epitaxial film. It has been reported that the FWHM of the (0002) plane reflects lattice distortion from screw dislocations and mixed dislocations, while that of the asymmetric (10-12) plane FWHM is associated with lattice distortion from edge dislocations and mixed dislocations. We have clearly seen that the low FWHM of GaN (0002) plane samples showed smoother surface morphology as measured by AFM. We found that the optimized growth temperature and buffer layers are playing critical role to obtain high quality GaN films. Further increase in growth temperature above $720{ }^{\circ} \mathrm{C}$ dramatically reduced the growth rate due to desorption of $\mathrm{GaN}$ or $\mathrm{Ga}$ from the growing surface. ${ }^{9}$
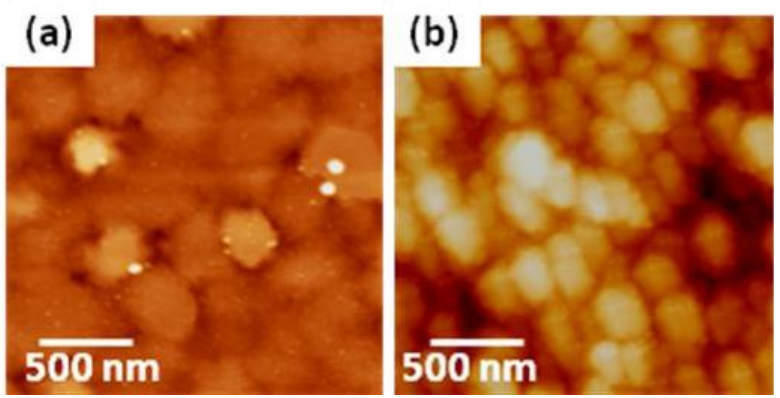

Fig. 4: AFM images of $\mathrm{GaN}$ films grown at $500{ }^{\circ} \mathrm{C}$ under high pressure nitrogen gas on sapphire (0001): (a) with nitridation, (b) without nitridation.

Fig. 5 shows a typical room temperature PL spectrum of $240 \mathrm{~nm}$ thin LMBE grown GaN film on nitridated sapphire. The PL spectrum has been recorded at different places of the sample by spatial movement of the sample in front of fixed source beam spot. Since the signal intensity does not change much from place to place, the intensities of the spectrum taken from the sample was plotted after averaging all statistical runs. This PL spectrum is acquired at a fixed excitation wavelength of $325 \mathrm{~nm}$. Two main emission peaks were observed as indicated by straight dashed line in the PL curve. The band edge transition peak centers at $369 \mathrm{~nm}$, corresponding to energy of $3.36 \mathrm{eV}$. The band gap of the hexagonal phase GaN film is nearly $3.4 \mathrm{eV}$ at room temperature. ${ }^{11}$ The HR-XRD and micro-Raman analysis shows that our GaN film grown on sapphire using LMBE technique has hexagonal phase. ${ }^{9}$ The band edge emission is different for GaN films grown by different growth techniques, kind of substrate, substrate temperature and buffer layers on substrates. For example, a band edge emission for MOCVD grown GaN sample is $3.51 \mathrm{eV}$ whereas it is $3.42 \mathrm{eV}$ for MBE grown samples. ${ }^{12}$ Cazzanelli et. $a l^{13}$ also observed the band edge emission at $3.36 \mathrm{eV}$ for a pulsed laser deposition (PLD) grown GaN films on sapphire. The origin of the band edge emission peak is well known from earlier experiments and the band edge emission region is mainly dominated by free exciton transition, donor-to-valence band transition and donorbound exciton transitions, among others. ${ }^{14-16}$ In addition, the thermal broadening, defect related disorder and impurities levels in the GaN film also increases the broadening of the band edge emission peak.

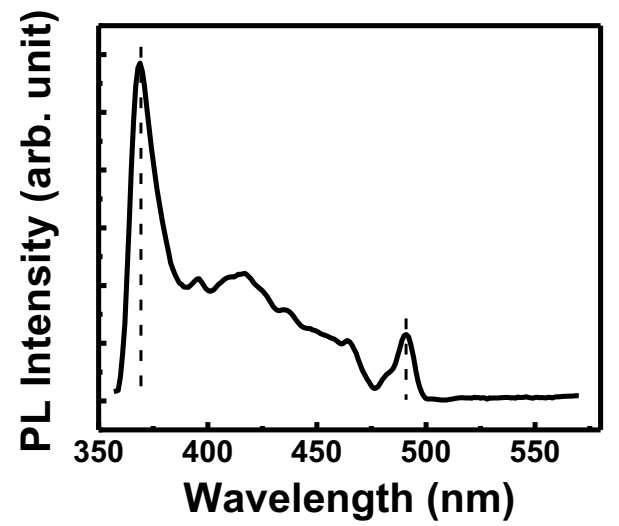

Fig. 5. A typical photoluminescence spectrum of GaN film grown on sapphire using LMBE technique.

Another peak in Fig. 5 corresponds at $2.52 \mathrm{eV}$ (wavelength $492 \mathrm{~nm}$ ) which could be related to the greenish blue luminescence. Generally, GaN film exhibits a mid gap level broad defect related PL peak, mainly attributed with yellow luminescence band, centered at $\sim 2.2-2.4 \mathrm{eV}$ as reported for GaN layers grown by various techniques. ${ }^{15,16}$ The origin of yellow band luminescence peak has been associated to various kinds of surface defects, dopant or impurities related transitions: transition from shallow donors (substitutional $\mathrm{O}$ and $\mathrm{C}$ complexes, $\mathrm{O}_{\mathrm{N}}$ and $\mathrm{C}_{\mathrm{N}}$ ) to deep acceptors $\left(\mathrm{Ga}\right.$ vacancies, $\mathrm{V}_{\mathrm{Ga}}$ ). For our LMBE grown GaN samples, we did not observe any yellow band PL peak. Even in earlier report, Sun et. $a l^{17}$ also did not observe any yellow band luminescence peak for GaN film grown on sapphire using liquid target PLD system. The absence of yellow luminescence peak in GaN film indicates the high crystalline quality of the film. The micro-Raman studies also complimented the high crystalline quality with a sharp 
characteristic peaks related to wurtzite c-plane GaN grown on sapphire using LMBE. The secondary ion mass spectroscopy measurements revealed a non-traceable level of oxygen background impurity in the GaN film mainly due to use of high pure HVPE grown GaN bulk target and UHV environment. ${ }^{9}$

\section{CONCLUSIONS}

We have grown epitaxial GaN films on pre-nitridated sapphire (0001) substrates at various growth temperatures ranging from 500 to $720{ }^{\circ} \mathrm{C}$ in high flux regime using a LMBE system. The FWHM of x-ray diffraction rocking curve of $\mathrm{GaN}$ film decreases with growth temperature from 1582 arc $\sec \left(500{ }^{\circ} \mathrm{C}\right)$ to $102 \operatorname{arc} \sec \left(720{ }^{\circ} \mathrm{C}\right)$, indicating the high crystalline quality growth at higher temperatures. The AFM studies also showed that the average grain size of the GaN film increases from $75-85 \mathrm{~nm}$ to flat $200-300 \mathrm{~nm}$ with increasing growth temperature from 500 to $720{ }^{\circ} \mathrm{C}$. At growth temperature of $700{ }^{\circ} \mathrm{C}$, we obtained that structural quality of $\mathrm{GaN}$ film is better (110 arc sec FWHM GaN (0002) plane) for high flux growth regime compared to low flux regime (FWHM 368 arc sec). We have also found that prenitridation on sapphire substrate helps to grow high quality smooth GaN film. The optical property measured by PL on the $\mathrm{GaN}$ film on sapphire shows that there is no yellow luminescence peak which indicates the growth of high quality film with minimal defects. These results show that the growth temperature, buffer layer and flux strongly influence the surface morphology and crystalline quality of the GaN films on sapphire.

\section{ACKNOWLEDGMENTS}

The authors are grateful to Prof. R.C. Budhani for the constant encouragement and support. The author would like to thank
Mr. Sandeep Singh for AFM measurements. This work was funded by Council of Scientific and Industrial Research (CSIR) under projects (NWP-25 and PSC0109).

\section{REFERENCES}

[1] S. Nakamura, M. Senoh, N. Iwasa, and S.I. Nagahama, Appl. Phys. Lett. 67, 1868 (1995).

[2] P. Ravadgar, R.H. Horng, and S.L. Ou, Appl. Phys. Lett. 101, 231911 (2012).

[3] C. Mion, J.F. Muth, E.A. Preble, and D. Hanser, Appl. Phys. Lett. 89, 092123 (2006).

[4] K. Yamane, M. Ueno, H. Furuya, N. Okada, and K. Tadatomo, J. Cryst. Growth 358, 1 (2012).

[5] A. Guillén-Cervantes, Z. Rivera-Álvarez, M. López-López, A. PoncePedraza, C. Guarneros, and V.M. Sánchez-Reséndiz, Appl. Surf. Sci. 258, 1267 (2011).

[6] D. Kapolnek, X.H. Wu, B. Heying, S. Keller, B.P. Keller, U.K. Mishra, S.P. DenBaars, and J.S. Speck, Appl. Phys. Lett. 67, 1541 (1995).

[7] M. Kumar, B. Roul, T.N. Bhat, M.K. Rajpalke, and S.B. Krupanidhi, Appl. Phys. Exp. 5, 085202 (2012).

[8] R.D. Vispute, V. Talyansky, R.P. Sharma, S. Choopun, M. Downes, T. Venkatesan, K.A. Jones, A.A. Iliadis, M.A. Khan, and J.W. Yang, Appl. Phys. Lett. 71, 102 (1997).

[9] S.S. Kushvaha, M. Senthil Kumar, K.K. Maurya, M.K. Dalai, and N.D. Sharma, AIP Advances 3, 092109 (2013).

[10] K. Uchida, A. Watanabe, F. Yano, M. Kouguchi, T. Tanaka, and S. Minagawa, J. Appl. Phys. 79, 3487 (1996)

[11] B. Monemar, Phys. Rev. B 10, 676 (1974).

[12] M.O. Manasreh and A.K. Sharma, Mater. Res. Soc. Symp. Proc. 395, 553 (1996).

[13] M. Cazzanelli, D. Cole, J.F. Donegan, J.G. Lunney, P.G. Middleton, K.P. O'Donnell, C. Vinegoni, and L. Pavesi, Appl. Phys. Lett. 73, 3390 (1998).

[14] M. H. Kim, Y. C. Bang, N. Man. Park, C. J. Choi, T. Y. Seong and S. J. Park, Appl. Phys. Lett. 78, 2858 (2001).

[15] D.O. Demchenko, I.C. Diallo, and M.A. Reshchikov, Phys. Rev. Lett. 110, 087404 (2013).

[16] M. A. Reshchikov and R. Y. Korotkov, Phys. Rev. B 64, 115205 (2001).

[17] X.W. Sun, R.F. Xiao, and H.S. Kwok, J. Appl. Phys. 84, 5776 (1998). 